\title{
Dynamics applications in the vehicle and two-wheeler accidents
}

\author{
Ming Ni \\ Department of Physics and Technology, Kunming University, Kunming 650214, China \\ kmxynm@163.com
}

Keywords: Statistical Analysis, Accident Analysis, Responsibility Identification, Collision Models, Uncertainty Analysis

\begin{abstract}
Conflicts between the parties have been increased due to multiple accidents between vehicles and two-wheelers. The main problem lies to the responsibility identification in the accident. Speed collision by applying to scientific and accurate accident reconstruction is an important part for the responsibility identification in an accident. Thus, the different forms of accident analysis is of great significance for improving the handling level in accidents. In the research on analysis methods in the accident between vehicles and bicycles, the braking traces, the throw distance models of the rider and the bike, as well as the throw distance fallout will be analyzed.
\end{abstract}

\section{Introduction}

Faced with complex and diverse traffic accidents, different types of accident investigation are being conducted around the world, such as the frontal collision, side collision, oblique collision of vehicles and other aspects of security. It provides the necessary guidance in making the traffic safety laws and regulations. At present, the research into the collision of the aspects is so well-conducted and systematic that the corresponding software has been developed to simulate, reconstruct and identify. For example, the EU and Japan have set up a two-wheeled traffic safety research institute in order to study the collision feature between two-wheelers and vehicles[1].

The United States, European Union and other advanced countries have developed their own traffic safety standards and regulations based on a lot of investigation and the actual vehicle collision on two-wheelers[2-4]. The main problem of accidents handling is to identify responsibility .Only in a reasonable and fair way to identify the responsibility of the parties can conflicts be resolved. Identifying the collision speed by applying scientific and accurate accident reconstruction is an important aspect of responsibility identification in the accidents $[5,6]$.

\section{The quantization and statistics of the collision location}

The deformation index (VDI-Vehicle Deformation Index)of the vehicle will be described, referring to the collision position between a vehicle and a two-wheeler. Divide the vehicle and the two-wheeler into 12-bit areas by the reference line of the longitudinal axis of a vehicle or a two-wheeler. The clockwise is positive. Divide the collision position as shown in Figure 1.

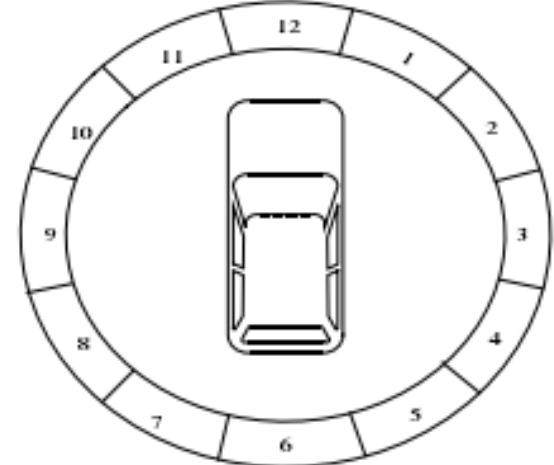

a) the division of vehicle collision position

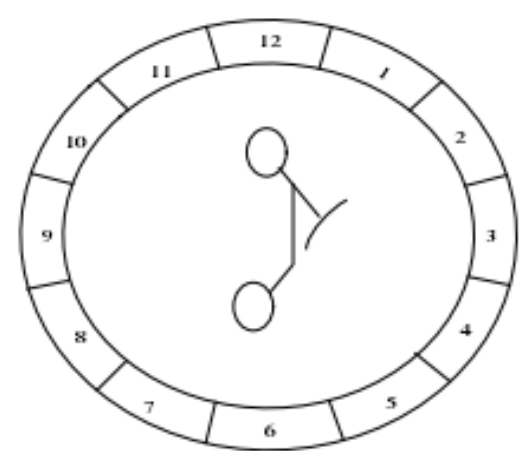

b) the division of a wheeler collision position Figure 1 the division of collision position 
In order to obtain the collision information of different areas of the 12 bit areas between the vehicle and the two-wheeler, the collision position distribution between vehicles and two-wheelers are studied in two types, as is shown in Figure 2.

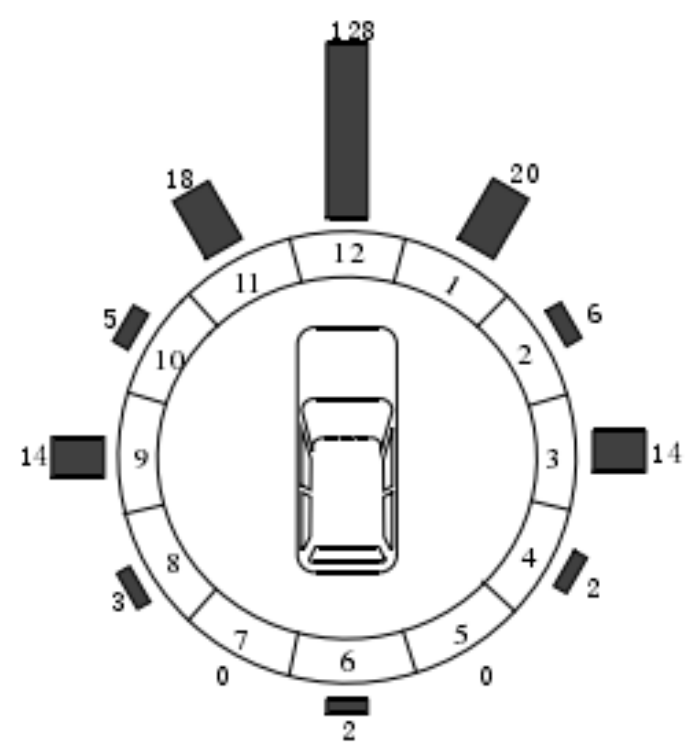

(a) the statistics of the vehicle collision location of the accident

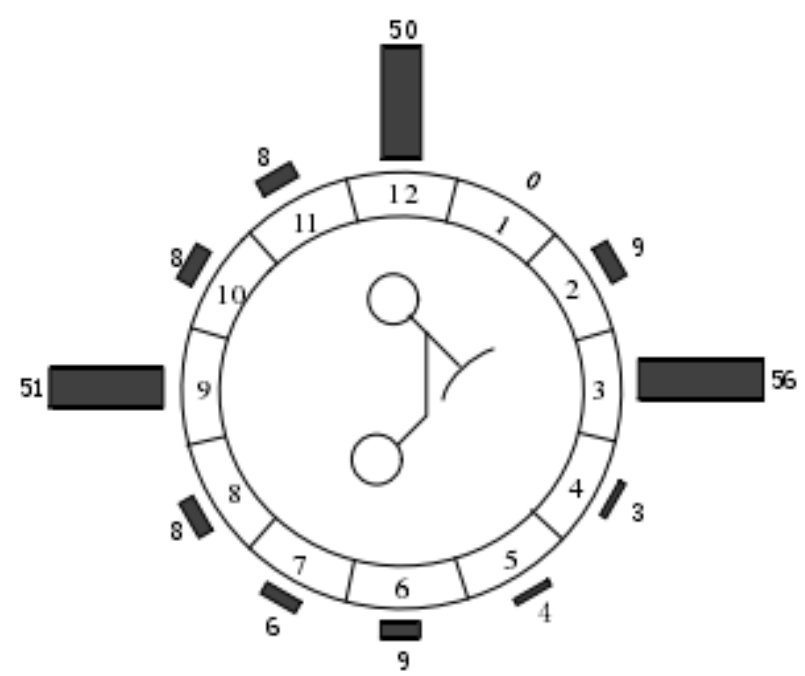

(b) the statistics of the two-wheeler collision location of the accident

Figure 2 the number of accidents of the collision position (Unit: case)

Statistics from the results in Figure 2 show that the maximum probability of an accident in a vehicle collision is Bit Area12, namely the frontal collision, which accounts for $60.38 \%$ of the total accidents, followed by Bit Area 1 and Bit Area 11. It means that the right front corner of the car and the left front corner crash the wheelers, accounting for separately $8.49 \%$ and $9.43 \%$ of the total number of the accidents. The bit areas of collision probability for a two wheeler is Bit Area 12, Bit Area 3 and Bit Area 9. So frontal collision and side crashes are the main types in two-wheeler accidents, accounting for separately $23.58 \%, 26.42 \%$ and $24.06 \%$ of the total number of the accidents

\section{The analysis method according to braking traces}

In an accident between the actual collison of vehicles and bicycles or electric bikes, the drivers take emergency braking after the vehicle crashes, which will leave braking traces on the surface of the road. The vehicle braking speed is solved according to the length of the braking drag:

The formula for calculation of the braking speed by using traditional methods is:

$$
v_{0}=\sqrt{2(\varphi \pm i) g S}
$$

$v_{0}$ is the braking initial velocity, unit: $\mathrm{m} / \mathrm{s} ; \varphi$ is the tire / road adhesion coefficient; $\mathrm{i}$ is the road gradient (\%); $\mathrm{S}$ is the length of the braking trace, the unit: $\mathrm{m}$.

Use Neptune model [7] for solving vehicle the braking speed:

$$
v_{0}=\sqrt{2(\varphi \pm i) g S}+\frac{1}{2}(\varphi \pm i) g t
$$

$\mathrm{t}$ is the braking coordinate time, taking $0.2 \mathrm{~s} ; \mathrm{g}$ is the gravitational acceleration, taking $9.8 \mathrm{~m} / \mathrm{s}$.

From the formulas of Model (1) and Model (2), when the length of the braking trace is fixed, the size of the friction coefficient determines the size of the output parameters. Therefore, it is necessary to reduce the error coefficient of adhesion to make calculations more accurate. The 
adhesion coefficient got by testing braking in a vehicle accident scene is closest to that when collision occurs.But the braking test is seldom done in the scene for security reasons and traffic order. The study results of Reference 7 shows that the calculation error in Model (1) is within 20\%, while the calculation error in Model (2) is within $10 \%$. So in most applications, Model(2) is more accurate than Model (1). The braking traces are made by people, vehicles, roads, environment and other factors. They have a certain nonlinear characteristics, its expression can be as follows:

$$
S=f(p, c, r, e)
$$

$\mathrm{S}$ represents the length of the braking trace; p represents the human factor, such as braking reaction time; c represents factors vehicles, such as the braking initial velocity, the breaking time, the size of the braking force, the function of ABS, and so on; r represents the road factors, such as the tire / ground adhesion coefficient; e represents environmental factors, such as dryness, horizon. As can be seen from equation (3), the braking initial velocity is one of the reasonsof making brakes. The simple use of the braking traces to reproduct braking initial velocity of the vehicle is a little difficult. Automotive theoretical analysis shows that the appearance of braking traces does not mean the beginning of the braking of a vehicle. The braking traces are not very obvious for the vehicles equipped with anti-lock devices (ABS) , and in many cases it is difficult to survey itstraces. Therefore, the conditions of people, vehicles, roads, and environment should be considered when the braking trace model is used, reducing errors during reproduction.

\section{The accident analysis methods based on the throw distance of the rider and the bike}

The collision mechanism between vehicles and two-wheeler is complex. It's difficult to accurately calculate the collision speed by using mathematical models. But the collision speed calculation formula which is within a certain error acceptance can be fitted out [8]. According to a large number of experimental studies and theoretical analysis, the study on relation of the throw distance of the rider and a bicycle between the collision speed of the vehicle can increase the solving path of the reconstruction of the collision speed.

37 real vehicle collision have been tested in the use of dummies and stationary bike fixed in front of the vehicles document. The relationship of the average throw distance between the rider as well as the bike and the collision vehicle if shown as follows:

the throw distance of the rider:

$$
S_{1}=0.033 v^{159}
$$

the throw distance of the bike:

$$
S_{2}=0.044 v^{157}
$$

$\mathrm{v}$ is the collision speed, $\mathrm{km} / \mathrm{h} ; S_{1}$ is the throw distance of the rider, $\mathrm{m} ; S_{2}$ is the throw distance of the bicycle, $\mathrm{m}$. Build accident model in which the front of the vehicle crashes the sideof the bicycle in the use of PC-CRASH software in Reference [9]. The relationship between the throw distance of the rider as well as the bicycle and is studied, whose model is:

the throw distance of the rider: $S_{1}=0.011 v^{1.367}$

the throw distance of the bike: $S_{2}=0.012 v^{1914}$

\section{Conclusion}

Based on the above analysis, it is practical to reconstruct the accident by means of the model of the throw distance of the rider and the bike. With simple formula, less input parameters, the stable model and calculating collision speed without depending on automotive braking traces, it has many advantages. To infer the collision speed of the vehicle is an important part in the accident analysis according to the scene information, such as braking drag, windshield fallout, the throw distance of the human body, etc. It is also the important basis of analyzing the cause of the accident and classifying the accident responsibility. 


\section{References}

[1] European Enhanced Vehicle-safety Committee Working group 8 on cycle and light-powered two-wheeler accidents. Cycle and light powered two-wheeler accidents. Proceedings of the Ninth IRCOBI Conference, Delft, September 1984, and Proceedings of the Tenth ESV Conference, Oxford, 1985.

[2] Preusser D.F., Williams A.F., Ulmer R.G. Analysis of fatal motorcycle crashes: crash typing [J]. Accident Analysis and Prevention, 1995, 27(6):845-851.

[3] Rasanen M., Summala H. Attention and expectation problems in bicycle-car collision: an in-depth study [J]. Accident Analysis and Prevention, 1998, 30:657-666.

[4] Simoncic M. Road accidents in Slovenia involving a pedestrian, cyclist or motorcyclist and a car [J]. Accident Analysis and Prevention, 1998, 30:147-156 .

[5] Yong $\mathrm{Lu}$ The Analyse of Issues about Domestic Motorcycle Traffic Safety [J] Guizhou University of Technology (Social Science), 2007, 5 (9): 126-130.

[6] Zhiqiang Liu, Peng Wang, Jianhua Zhang Accident Reconstruction Technology Research status and development trend [J] China Safety Science Journal, 2007, 4 (17): 16-20 .

[7] Neptune, J.A., Flynn, J.E., Chavez, P.A. and Underwood, H.W. Speed from Skids: A Modern Approach [J]. SAE, paper 950354, 1995.

[8] Hongguo Xu, Jian Su, Wei Gao, Study on the Speed Confirmation of Bike Collision [J] Chinese Journal of Highway, 1996, 9 (3): 98-103.

[9] Qingfeng Lin, Hongguo Xu, Bo Chen The Throw Distance Model of Pedestrians, Cyclists and Bikes in the Traffic Accidents [J] Transportation Systems Engineering and Information Technology, 2006, 6 (6): 73-78. 